\title{
Probable Herbal Treatment of Leprosy
}

\author{
Kavita Niranjan ${ }^{1}$, Dr.Suhasini Bhatnagar ${ }^{2}$ \\ '(,Scientific Consultant at MB life sciences,Delhi) \\ ${ }^{2}$ (Biotechnology department,Mewar University, Ghaziabad)
}

\begin{abstract}
The causative agent of leprosy Mycobacterium leprae has a lipid rich cell wall which contributes to virulence and antibiotic resistance. Acyl Coenzyme A Synthetase catalyzes the step fatty acid activation during fatty acid synthesis. Analysis of genes FadD26 and FadD13 of Mycobacterium leprae and Mycobacterium tuberculosis respectively demonstrated that melonate can be consider as FadD26 ligand and beetroot source of melonate can be use as an herbal treatment of leprosy.
\end{abstract}

Keywords: Acyl Coenzyme A synthetase, Beetroot, FadD genes, Fatty acid synthtase system, lipid rich cell wall, Mycobacterium Leprae, Melonate

\section{Introduction}

Leprosy, a major source of morbidity in developing countries, is a chronic infectious disease caused by the obligate intracellular bacterium Mycobacterium leprae [1,2]. According to the system of classification of Ridley and Jopling (1966), leprosy patients show two major manifestations of the disease, designated as lepromatous leprosy (LL) and tuberculoid leprosy (TL) [1]. TL is observed in patients with good T-cell mediated (Th1) immunity and is characterized by granuloma formation and death of Schwann cells (SCs) leading to loss of myelin sheath and nerve destruction [3, 4]. TL shows only few lesions, and bacilli can rarely be identified. Patients with poor T-cell mediated immunity show the lepromatous-type leprosy (LL). LL leads to massive bacterial load inside host cells specially SCs and macrophages [ $\underline{3}, \underline{5}-\underline{7}]$. The lesions of TL and LL types are named as T-lep and L-lep lesions, respectively, but damage of the nerves is observed in most of the cases of both types [7].

Lepromatous leprosy exhibits multiple lesions of the skin, eyes, nerves, and lymph nodes, which are characterized by tumor-like accumulations of foamy macrophages. The foamy macrophages are fully packed with lipid droplets (LDs) and contain high numbers of lepra bacilli. These aggregations of foamy macrophages expand slowly and disfigure the body of the host [8].

M.Leprae has a small genome (3.2 Mb). The obligate intracellular organism shows a moderate genome degradation and several genes are absent when compared with other Mycobacterial species. Due to the gene loss M.Leprae is strongly dependent on the host for basic metabolic functions [9, 10]. Macrophages infected with M.Leprae contain oxidized host lipids and it has been observed that M.Leprae up regulates 13 host lipid metabolism genes in T-lep lesions and 26 in L-lep lesions. The oxidized lipids inhibit innate immune responses and thus seem to be an important virulence factor for the organism [11].

Mycobacteria have outstanding mechanisms to escape from elimination and have a high degree of intrinsic resistance to most antibiotics, chemotherapeutic agents and immune eradication [12, 13]. One major obstacle for host defence mechanisms and therapeutic intervention is the robust, lipid-rich cell wall, which is unique among prokaryotes [12,14]. The major problem in combating Mycobacterial diseases is that these bacteria produce unusual cell walls with low permeability, making them resistant to therapeutic agents. Successful antimycobacterial therapy includes the use of agents that inhibit the biosynthesis of cell-wall components. The widely used drug isoniazid inhibits the biosynthesis of mycolic acids $[15,16,17]$, one of the major cell-wall components. With the widespread appearance of resistance to such drugs, new drug targets must be identified to combat mycobacterial infections. One of the unusual features of the Mycobacterial cell wall is that it is composed of many unique lipids constituting $60 \%$ of the wall. An understanding of the biochemistry and molecular genetics of the cell-wall lipids would provide a rational basis for seeking drugs targeted at the production of such unique Mycobacterial lipids.

\subsection{The wide variety of lipids in Mycobacteria}

Mycobacteria have an extremely wide diversity of lipids that are quite unlike the usual bacterial cellular lipids which are also present in Mycobacteria $[18,19]$. As the biochemistry and molecular genetics of this organism need to be understood in the context of production of such lipids, a brief look at their diversity is useful. In addition to the usual fatty acids found in membrane lipids, Mycobacteria have a wide variety of very long-chain saturated (C18-C32) and mono unsaturated (up to C26) n-fatty acids. The occurrence of $\alpha$-alkyl $\beta$ hydroxy very long-chain fatty acids, i.e. mycolic acids, is a hallmark of Mycobacteria and related species. 
Mycobacterial mycolic acids are the largest (C70-C90) with the largest $\alpha$-branch (C20-C25). The main chain contains one or two double bonds, cyclopropane rings, epoxy groups, methoxy groups, keto groups or methyl branches. Such acids are major components of the cell wall, occurring mostly esterified in clusters of four on the terminal hexa-arabinofuranosyl units of the major cell-wall polysaccharides called arabinogalactans. They are also found esterified to the 6 and 6 positions of trehalose to form 'cord factor'. Small amounts of mycolate are also found esterified to glycerol or sugars such as trehalose, glucose and fructose depending on the sugars present in the culture medium.

Mycobacteria contain a wide variety of methyl-branched fatty acids. These include 10-methyl C18 fatty acid (tuberculostearic acid found esterified in phosphatidyl inositide mannosides), 2,4-dimethyl C14acid and mono-, di- and trimethyl-branched $\mathrm{C} 14$ to $\mathrm{C} 25$ fatty acids found in trehalose-containing lipooligosaccharides, trimethyl unsaturated C27 acid (phthienoic acid), tetramethyl-branched C28-C32 fatty acids (mycocerosic acids) and shorter homologues found in phenolic glycolipids and phthiocerol esters, and multiple methyl-branched phthioceranic acids such as heptamethyl-branched C37 acid and oxygenated multiple methyl-branched acids such as 17-hydroxy-2,4,6,8,10,12,14,16-octamethyl C40 acid found in sulpholipids. In addition, mycocerosic acids and other branched acids are esterified to phthiocerol and phenolphthiocerol and their derivatives.

\subsection{Biosynthesis of n-fatty acids}

Both multifunctional fatty acid synthase (FAS) (type I), usually found in eukaryotes, and multicomponent FAS (type II), usually found in prokaryotes, have been found in Mycobacteria. The multifunctional synthase catalyses not only the synthesis of C16and C18 fatty acids, the normal products of de novo synthesis, but also elongation to produce C24 and C26 fatty acids [20, 21].

Mycobacterial FAS is larger than the other multifunctional synthases. Presumably, this synthase has unusual acyl transferase and ketoacyl synthase domains that could account for the elongating activity of this synthase [22]. The outlined diagrammatic process is shown below:

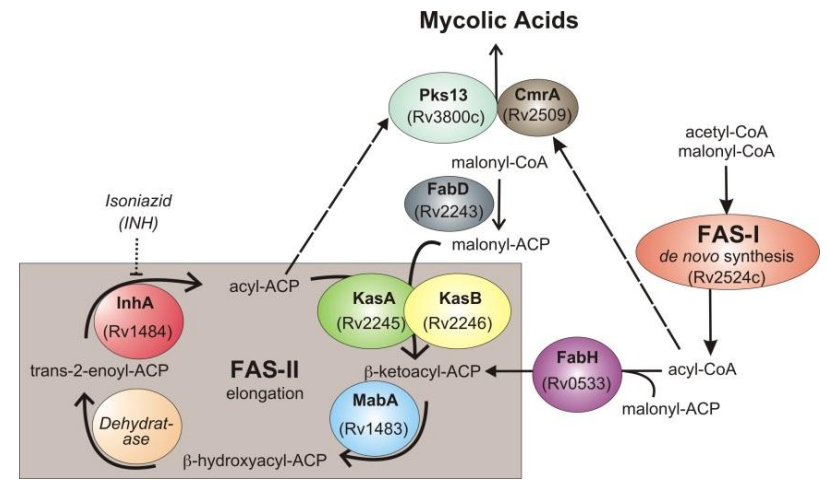

\subsection{Acyl Co A Synthetase}

Activity of Acyl Co A synthetase in fatty acid biosynthesis has been demonstrated in Mycobacteria. This is identified as one of the important enzyme involve in biosynthesis of fatty acids in Mycobacteria. In Mycobacterium leprae Br4923, this enzyme is encoded by gene FadD26, which activates fatty acids by binding to coenzyme $\mathrm{A}$ and the reaction, is -

octanoate + ATP + coenzyme A $\Leftrightarrow$ octanoyl-CoA + AMP + diphosphate [23]

Fad genes also found in other mycobacterial species like M.tuberculosis .this organism contain FadD13 gene encoded acyl Co A Synthetase. In M. tuberculosis melonate is one of the ligand of this enzyme [24]. By blocking of enzyme active site through melonate we can block activity of acyl Co A synthetase enzyme and further fatty acid activation.

To prove this hypothesis we used Blast, blast gave $100 \%$ sequence similarity between FadD26 and FadD13 both encoded acyl Co A Synthetase.

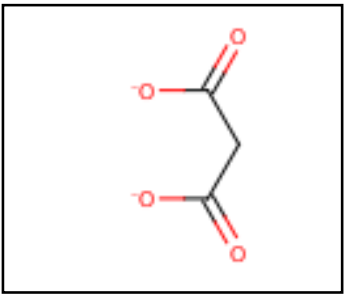

Fig.1 melonate ion 
As compared to the synthesis route in Humans is quite different and in summary it is:

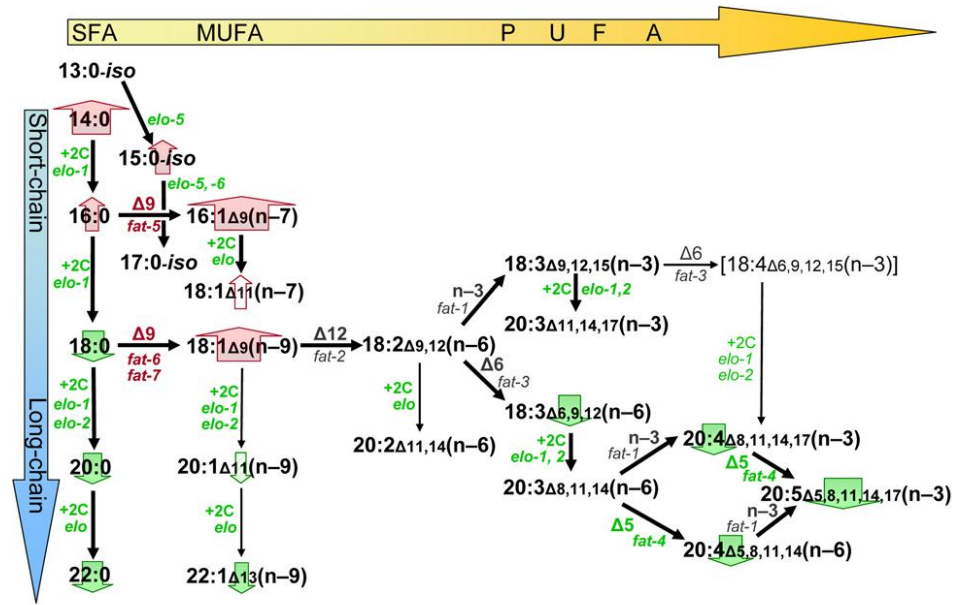

Blast between FadD26 and FadD13

\section{Results}

Basic Local Alignment Search Tool

ZB/ BLAST/ blastn suite-2sequences/ Formatting Results - 7A4HNZVJ11N

Eormatting options

Download

Blast 2 sequence

ucle otide Sequence (1512 letters)

RID TA4HNZVJ11N (Expires on 11-03 21:54 pm)

Query ID $|c| \mid 23547$
Description

Description None

Molecule type nucleic acid

Subject ID $|c| \mid 23549$

Molecule type nucleic acid

$\begin{aligned} \text { Subject Length } & 1752 \\ \text { Program } & \text { BLASTN 2.2.28 }\end{aligned}$

Graphic Summary

Distribution of 12 Blast Hits on the Query Sequence

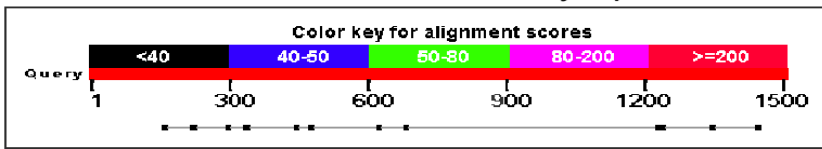

Fig. 2 blast result

\section{Descriptions}

\begin{tabular}{|l|l|l|l|ll|l|}
\hline Description & Max score & Total score & Query cover & E value & Ident & Accession \\
\hline None provided & 27.6 & 260 & $9 \%$ & 0.013 & $100 \%$ & 23549 \\
\hline
\end{tabular}

Fig.3 descriptions

\section{Discussion}

From the observation using the Bioinformatics software protein data base and Blast suggest that melonate will inhibit the activity of Acyl Co A Synthetase enzyme and as a result M. leprae should not able to use this enzyme and synthesis of fatty Acids. This must lead to a porus cell wall by which the external particle can enter inside and the bacteria in early stages should die because of leakage.

\section{Conclusion}

The lipid rich cell wall in M. leprae plays an important role in pathogenesis. Several enzymes of the mycobacterial lipid-biosynthesis are regarded as targets for new drugs and this study shown that If research 
work of Acyl Co A Synthetase enzyme is encouraged, this will be a vital step to find the life saving drug in case of leprosy.

\section{Acknowledgement}

We acknowledge the help of MB Life sciences, at Pritampura, New Delhi who supported us wisely during the study. We wish to thank them for the support.

\section{References}

[1]. D. S. Ridley and W. H. Jopling, "Classification of leprosy according to immunity. A five-group system," International Journal of Leprosy and Other Mycobacterial Diseases, vol. 34, no. 3, 1966, pp. 255-273.

[2]. $\quad$ WHO, "WHO Leprosy Today," WHO, 2010.

[3]. C. L. Cosma, D. R. Sherman, and L. Ramakrishnan, "The secret lives of the pathogenic mycobacteria," Annual Review of Microbiology, vol. 57, 2003, pp. 641-676.

[4]. D. M. Scollard, "Endothelial cells and the pathogenesis of lepromatous neuritis: insights from the armadillo model," Microbes and Infection, vol. 2, no. 15, 2000, pp. 1835-1843.

[5]. K. A. Mattos, F. A. Lara, V. G. C. Oliveira et al., "Modulation of lipid droplets by Mycobacterium leprae in Schwann cells: a putative mechanism for host lipid acquisition and bacterial survival in phagosomes," Cellular Microbiology, vol. 13, no. 2, 2011, pp. 259-273.

[6]. D. M. Scollard, L. B. Adams, T. P. Gillis, J. L. Krahenbuhl, R. W. Truman, and D. L. Williams, "The continuing challenges of leprosy," Clinical Microbiology Reviews, vol. 19, no. 2, 2006, pp. 338-381.

[7]. V. D. Vissa and P. J. Brennan, "Impact of the Mycobacterium leprae genome sequence on leprosy research," in Genomics of GCRich Gram-Positive Bacteria, A. Danchin, Ed., Band 2, 2002, pp. 85-118, Caister Academic Press,.

[8]. D. S. S. Raphael Rubin and R. Emanuel, Eds., Rubin's Pathology: Clinicopathologic Foundations of Medicine, Lippincott Williams \& Wilkins, 2007.

[9]. Cosma C. L., Sherman D. R., Ramakrishnan L. The secret lives of the pathogenic mycobacteria. Annu. Rev. Microbiol.57, 2003, 641-676.

[10]. Hagge D. A., Oby Robinson S., Scollard D., McCormick G., Williams D. L. A new model for studying the effects of Mycobacterium leprae on Schwann cell and neuron interactions. J. Infect. Dis., 186, 2002, 1283-1296.

[11]. Cruz D., Watson A. D., Miller C. S., Montoya D., Ochoa M. T., Sieling P. A., Gutierrez M. A., Navab M., Reddy S. T., Witztum J. L., Fogelman A. M., Rea T. H., Eisenberg D., Berliner J., Modlin R. L. Host-derived oxidized phospholipids and HDL regulate innate immunity in human leprosy. J. Clin. Invest, 118, 2008, 2917-2928.

[12]. Brennan P. J., Nikaido H. The envelope of mycobacteria. Annual review of biochemistry, 64, 1995, $29-63$.

[13]. Coker R. J. Review: multidrug-resistant tuberculosis: public health challenges. Tropical medicine \& international health : TM \& IH, 9, 2004, 25-40.

[14]. Barry C. E., 3rd, Lee R. E., Mdluli K., Sampson A. E., Schroeder B. G., Slayden R. A., Yuan Y. Mycolic acids: structure, biosynthesis and physiological functions. Progress in lipid research , 37, 1998, 143-179.

[15]. Besra, G.S., and Chatterjee, D. Lipids and carbohydrates of Mycobacterium tuberculosis. InTuberculosis. Pathogenesis, Protection, and Control. Bloom, B.R. (Ed.). (Washington, DC:American Society for Microbiology, 1994) pp. 285-306.

[16]. Dessen, A., Que'mard, A., Blanchard, J.S., Jacobs, Jr, W.R., and Sacchettini, J.C. Crystal structure and function of the isoniazid target of Mycobacterium tuberculosis. Science267, 1995, 1638-1641.

[17]. Wheeler, P.R., and Anderson, P.M. Determination of the primary target for isoniazid in mycobacterial mycolic acid biosynthesis with Mycobacterium aurum Ap .Biochem J318, 1996, 451-457.

[18]. Brennan, P.J, and Nikaido, H. The envelope of mycobacteria. Annu Rev Biochem64, 1995, $29-63$.

[19]. Minnikin, D.E. Lipids: complex lipids, their chemistry, biosynthesis and roles. InThe Biology of the Mycobacteria: Vol. 1.44. Physiology, Identification and Classification. Ratledge, C., and Stanford, J. (Eds). (New York: Academic Press, 1982) pp. 95-184

[20]. Wood, W.I., Peterson, D.O., and Bloch, K. Mycobacterium smegmatis fatty acid synthase. A mechanism based on steady state rates and product distributions. J Biol Chem252, 1977, 5745-5749.

[21]. Kikuchi, S., Rainwater, D.L., and Kolattukudy, P.E. Purification and characterization of an unusually large fatty acid synthase from Mycobacterium tuberculosisvar. bovisBCG. Arch Biochem Biophys295, 1992, 318-326.

[22]. Fernandes, N.D., and Kolattukudy, P.E. Cloning, sequencing and characterization of a fatty acid synthaseencoding gene fromMycobacterium tuberculosisvar.bovis BCG.Gene170, 1996, 95-99.

[23]. http://biocyc.org/MLEP561304/NEW-IMAGE?type=ENZYME\&object=GJP6-2718-MONOMER

[24]. http://www.pdb.org/pdb/explore/explore.do?structureId=3R44 